\title{
Pion Condensation by Rotation in a Magnetic Field
}

\author{
Yizhuang Liu* and Ismail Zahed ${ }^{\dagger}$ \\ Department of Physics and Astronomy, Stony Brook University, Stony Brook, New York 11794-3800, USA
}

(Received 25 October 2017; revised manuscript received 4 December 2017; published 17 January 2018)

\begin{abstract}
We show that the combined effects of a rotation plus a magnetic field can cause charged pion condensation. We suggest that this phenomenon may yield to observable effects in current heavy ion collisions at collider energies, where large magnetism and rotations are expected in off-central collisions.
\end{abstract}

DOI: 10.1103/PhysRevLett.120.032001

Introduction.-The combined effects of rotations and magnetic fields on Dirac fermions are realized in a wide range of physical settings, ranging from macroscopic spinning neutron stars and black holes [1] all the way to microscopic anomalous transport in Weyl metals [2]. In any number of dimensions, strong magnetic fields reorganize the fermionic spectra into Landau levels, each with a huge planar degeneracy that is lifted when a parallel rotation is applied. The past decade has seen great interest in the chiral and vortical effects and their relationship with anomalies (see Ref. [3] and references therein).

Perhaps a less well-known effect stems from the dual combination of a rotation and a magnetic field on free or interacting Dirac fermions. Recently, it was noted that this dual combination could lead to novel effects for composite fermions at half filling in $1+2$ dimensions under the assumption that they are Dirac fermions [4], and more explicitly for free and interacting Dirac fermions in $1+3$ dimensions [5-7]. Indeed, when a rotation is applied along a magnetic field, the charge density was observed to increase in the absence of a chemical potential. A possible relationship of this phenomenon to the Chern-Simons term in odd dimensions and to the chiral anomaly in even dimensions was suggested. Recently, there have been a few studies along these lines using effective models of the NJL type in $1+3$ dimensions, where the phenomenon of charge density enhancement was also confirmed with new observations [6-8].

Current heavy ion collisions at collider energies in noncentral collisions involve large angular momenta in the range $10^{3}-10^{5} \hbar[9,10]$. Recently, STAR reported a large vorticity with $\Omega \sim(9 \pm 1) 10^{21} \mathrm{~s}^{-1} \sim 0.05 m_{\pi}$, by measuring the global polarization of $\Lambda$ and $\bar{\Lambda}$ in off-central Au-Au collisions in the Beam Energy Scan program [11]. During

Published by the American Physical Society under the terms of the Creative Commons Attribution 4.0 International license. Further distribution of this work must maintain attribution to the author(s) and the published article's title, journal citation, and DOI. Funded by SCOAP ${ }^{3}$. the prompt part of the collision, large magnetic fields $B \sim m_{\pi}^{2}$ are expected [12]. In this Letter, we show that the combined effects of magnetism plus rotation can induce a pion superfluid phase in off-central heavy ion collision. This superfluid phase may be at the origin of the large multipion correlations reported by ALICE [13], as is also suggested by a recent nonequilibrium study [14].

In this Letter, we first illustrate the basic mechanism at the origin of pion condensation. Next, we quantize the Klein-Gordon equation in a moving frame. We then make explicit the requirements for pion condensation for a charge-neutral system, estimate its amount in current heavy ion collisions at collider energies, and finally end with our conclusions.

Mechanism.-In the presence of a fixed magnetic field in the $+z$ direction, $\mathbf{B}=B \hat{z}$, the charged $\pi^{ \pm}$pion spectrum is characterized by highly degenerate Landau levels (LLs):

$$
E_{n p}=\left(|e B|(2 n+1)+p^{2}+m_{\pi}^{2}\right)^{\frac{1}{2}}
$$

with $p$ being the pion momentum along the 3 direction, each with a degeneracy $N=|e B| S / 2 \pi$, with $S=\pi R^{2}$ being the area of the plane transverse to $B$. We will assume that the magnetic length $l_{M}=1 / \sqrt{|e B|} \ll R$ for the LL to fit within $S$. In the circular gauge, the degeneracies of the LLs are identified with the eigenstates of the $z$ component of the angular momentum in position space. They are labeled by $l$, which enters the azimuthal wave function as $e^{i l \varphi}$ with the restriction $-n \leq l \leq N-n$, where $n$ labels the LL. For the lowest Landau level (LLL) with $n=0, l$ has a fixed sign, since $0 \leq l \leq N$. After quantization, the angular momentum for positive charged particles is $l$, and that for negative charged particles is $-l$. This means that in the LLL, the $\pi^{+}$ spins along the magnetic field, while the $\pi^{-}$spins opposite to the magnetic field as illustrated in Fig. 1.

When a rotation $\Omega$ along the magnetic field is applied, it causes the spectrum to shift linearly. Throughout, we will consider the parallel case with $\vec{\Omega} \cdot \vec{B}>0$ unless specified otherwise. With this in mind, and in the rotating frame, 


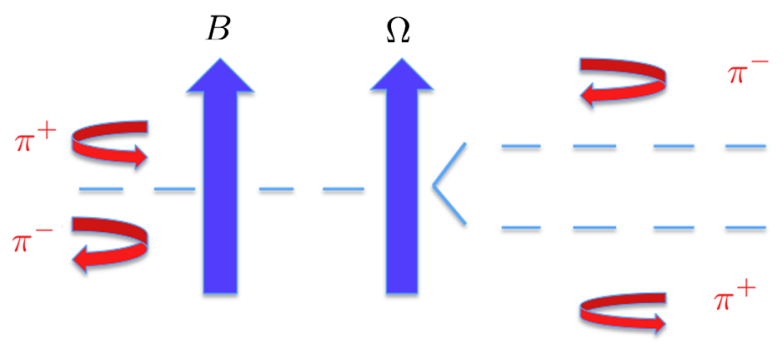

FIG. 1. Under the action of an external magnetic field $B$, the $\pi^{ \pm}$ undergo opposite rotations in the lowest Landau level (LLL), which is degenerate. The action of a parallel rotation $(\vec{\Omega} \cdot \vec{B}>0)$ lifts the degeneracy in the LLL. The energy of the $\pi^{+}$shifts down and splits away from the energy of the $\pi^{-}$that shifts up. The action of an antiparallel rotation $(\vec{\Omega} \cdot \vec{B}<0)$ exchanges the roles of $\pi^{+}$and $\pi^{-}$.

$$
E_{n p} \rightarrow E_{n p}-\Omega L_{z} \equiv E_{n p}-j \Omega l,
$$

with $j=+1$ for positively charged pions (particles) and $j=-1$ for negatively charged pions (antiparticles). As a result, the degeneracy of each LL is lifted. In particular, the $\pi^{+}$in the LLL splits down, and the $\pi^{-}$in the LLL splits up, as also illustrated in Fig. 1. Since the chargeless pions $\pi^{0}$ are unaffected by the magnetic field, their rotational shift averages out. Also, we note that causality requires $v=$ $\Omega R \leq 1$ [7], which, together with the magnetic length constraint (see above), translates to $l_{M} \ll R<1 / \Omega$.

The mechanism of $\pi^{ \pm}$splitting by a rotation parallel to a magnetic field in the LLL can cause $\pi^{+}$pion condensation. Indeed, in the shifted spectrum (2), the combination $\mu_{l}=$ $\Omega l$ plays the role of a chemical potential for $\pi^{+}$and $-\mu_{l}=$ $-\Omega l$ for $\pi^{-}$, in much the same way as noted for fermionic particles and antiparticles in the $\operatorname{LLL}[1,7,15,16]$. Therefore, when $\mu_{N}=N \Omega$ apparently exceeds the $\pi^{+}$ effective mass in the LLL, $m_{0}=\sqrt{e B+m_{\pi}^{2}}$, but it is still below the $\pi^{+}$effective mass in the first LL with $n=1$, the LLL $\pi^{+}$may Bose-condense.

Klein-Gordon equation in rotating frame.-To support Eq. (2), we now give a detailed derivation of the quantization of the Klein-Gordon equation in a rotating frame both for an infinite and for a finite volume $V=S L$. For that, we consider the metric for a rotating frame in $1+3$ dimensions with mostly negative signature $(+,-,-,-)$ :

$d s^{2}=\left(1-\Omega^{2} \rho^{2}\right) d t^{2}+2 y \Omega d x d t-2 x \Omega d y d t-d r^{2}$.

The comoving frame is defined as $e_{a}=e_{a}^{\mu} \partial_{\mu}$, with $\left(e_{0}, e_{1}, e_{2}, e_{3}\right)=\left(\partial_{t}+y \Omega \partial_{x}-x \Omega \partial_{y}, \partial_{x}, \partial_{y}, \partial_{z}\right)$. Now, consider a constant magnetic field in the $z$ direction, $B \hat{z}$, as described by the circular vector potential $A_{R \mu}=$ $B\left(0, y_{R} / 2,-x_{R} / 2,0\right)$ in the rest frame sublabeled by $R$. The coordinate transformation to the rotating frame $r_{R}=$ $r, t_{R}=t, \theta_{R}=\theta+\Omega t$ allows the rewriting of the vector potential in the rotating frame as

$$
A_{\mu}=B\left(-\Omega r^{2} / 2, y / 2,-x / 2,0\right) .
$$

In the rotating frame, there is, in addition to the magnetic field $B \hat{z}$, an induced electric field $\vec{E}=\Omega B \vec{r}$. This is expected from a Lorentz transformation from the fixed frame with $B \hat{z}$ to the comoving frame with $B \hat{z}$ and $\vec{E}$.

A charged scalar field $\Pi$ in the rotating frame and subject to the vector potential (4) is characterized by the Lagrangian

$\mathcal{L}=\left|\left(D_{t}+y \Omega D_{x}-x \Omega D_{y}\right) \Pi\right|^{2}-\left|D_{i} \Pi\right|^{2}-m_{\pi}^{2} \Pi^{\dagger} \Pi$,

with the long derivative $D_{\mu}=\partial_{\mu}+i e A_{\mu}$. We now note the identity

$$
D_{t}+y \Omega D_{x}-x \Omega D_{y}=\partial_{t}+y \Omega \partial_{x}-x \Omega \partial_{y} .
$$

The electric field following from Eq. (4) cancels out. The comoving frame corresponds only to a frame change with no new force expected. With this in mind, the equation of motion for the charged field in the rotating frame is

$$
-\left(\partial_{t}+y \Omega \partial_{x}-x \Omega \partial_{y}\right)^{2} \Pi-D_{i}^{\dagger} D_{i} \Pi-m_{\pi}^{2} \Pi=0 .
$$

In the infinite-volume case, we solve Eq. (7) using the algebraic ladder construction with

$$
\begin{aligned}
a & =\frac{i}{\sqrt{2 e B}}\left(D_{x}+i D_{y}\right), \\
b & =\frac{1}{\sqrt{2 e B}}\left(2 \partial+\frac{e B}{2} \bar{z}\right) .
\end{aligned}
$$

Choosing the positive $z$ direction to be that for which $e B$ is positive yields the operator identities

$$
\begin{aligned}
D_{x}^{\dagger} D_{x}+D_{y}^{\dagger} D_{y} & =e B\left(2 a^{\dagger} a+1\right), \\
L_{z} & =i\left(-x \partial_{y}+y \partial_{x}\right)=b^{\dagger} b-a^{\dagger} a .
\end{aligned}
$$

The general stationary solution to Eq. (7) is of the form $\Pi=e^{i p z-i E t} f$, with $f$ solving

$$
\left(E+\Omega L_{z}\right)^{2} f=\left(m_{\pi}^{2}+p^{2}\right) f+e B\left(2 a^{\dagger} a+1\right) f .
$$

The normalizable solutions form a tower of LLs

$$
\begin{aligned}
f_{m n} & =\frac{1}{\sqrt{m ! n !}}\left(a^{\dagger}\right)^{n}\left(b^{\dagger}\right)^{m} f_{00}, \\
\left(E_{m n}+\Omega(m-n)\right)^{2} & =e B(2 n+1)+m_{\pi}^{2} \equiv E_{n}^{2}
\end{aligned}
$$

with $f_{00} \sim e^{-(e B / 4)\left(x^{2}+y^{2}\right)}$ as the LLL. For the LL to fit in a volume $V=L S$, we need $m, n \leq N$. The quantized charged field $\Pi$ in the rotating frame is 


$$
\Pi=\int \frac{d p}{2 \pi} \sum_{n m} \frac{f_{m n}}{\sqrt{2 E_{n}}}\left(a_{n m p} e^{-i E^{+} t+i p z}+b_{n m p}^{\dagger} e^{i E^{-} t-i p z}\right),
$$

with the bosonic canonical rules

$$
\left[b_{n m p}, b_{n^{\prime} m^{\prime} p^{\prime}}^{\dagger}\right]=\left[a_{n m p}, a_{n^{\prime} m^{\prime} p^{\prime}}^{\dagger}\right]=2 \pi \delta_{n n^{\prime}} \delta_{m m^{\prime}} \delta\left(p-p^{\prime}\right) .
$$

The particle state created by $a_{n m p}^{\dagger}$ has energy $E^{+}=$ $E_{n}-\Omega(m-n)$, charge $+e$, and orbital angular momentum in the $z$ direction as $m-n$. The antiparticle state created by $b_{n m p}^{\dagger}$ has energy $E^{-}=E_{n}+\Omega(m-n)$, charge $-e$, and orbital angular momentum in the $z$ direction as $-m+n$. Therefore, the energy relationship between the rotating frame and the rest frame is $E=E_{R}-\Omega L_{z}$. This is in agreement with Eq. (2), where we have set $l=m-n$ and defined $L_{z}=j l$ with $j=+1$ for the particle or positive charge state and $j=-1$ for the antiparticle or negative charge state.

In a finite volume, Eq. (7) can be solved using instead the circular wave functions with zero boundary conditions:

$f_{l}(r, \theta)=e^{i l \theta} r^{|l|} e^{-\left(e B r^{2} / 4\right)} F_{1}\left(-a,|l|+1, \frac{e B r^{2}}{2}\right)$,

where ${ }_{1} F_{1}$ is a hypergeometrical function with the parameter

$-a(l)=\frac{1}{2}(|l|-l+1)-\frac{1}{2 e B}\left((E+\Omega l)^{2}-p^{2}-m_{\pi}^{2}\right)$.

Thus, for positive angular momentum states, we have

$$
\begin{aligned}
(E+\Omega l)^{2} & =p^{2}+m_{\pi}^{2}+e B(2 a(l)+1), \\
{ }_{1} F_{1}\left(-a(l), l+1, \frac{e B R^{2}}{2}\right) & =0 .
\end{aligned}
$$

The zero of the hypergeometric function fixes $a(l)$, and therefore the LL for a finite volume. For example, for $N=$ 25 we have $l=20$, and $a_{\min }(20)=0.43$, for which the energy is $E=\sqrt{m_{\pi}^{2}+1.86 e B}-20 \Omega$. For $e B=m_{\pi}^{2}$, the threshold rotation is $\Omega_{c}=(1.69 / 20) \sqrt{e B}$. Note that for $N=25$, we have $R=\sqrt{50 / e B}$, and the luminal constraint is still fulfilled, since $\Omega_{c} R=0.59<1$. For $N=100$, we have $l=84$, and $a_{\min }(84)=0.18$, for which the energy is $\sqrt{m_{\pi}^{2}+1.36 e B}-84 \Omega$ with the threshold $\Omega_{c} R=0.25$. For $N=1000$, we have $l=935$ and $a_{\min }(935)<0.1 e B$. Thus, as $N$ goes to infinity, the state with the lowest energy will approach $l=N$, and our approximation in the main text becomes more precise. Note that for exactly $l=N-1$, we always have $a_{\min }(N-1)=1$, and the energy for such a state is $\sqrt{m_{\pi}^{2}+3 e B}-(N-1) \Omega$.
Pion condensation.-For a fixed and isolated volume $V=S L$ with no charge allowed to flow in or out, strict charge conservation in the comoving frame is achieved by introducing a charge chemical potential $\mu$, in addition to the induced chemical potential $j \Omega l$ by rotation. (For an open volume discussion, see Ref. [16] and references therein). For the LLL, charge conservation requires that the number of $\pi^{ \pm}$in $V$ be equal at any temperature

$$
\begin{aligned}
& \sum_{l=0}^{N} \int \frac{d p}{2 \pi} \frac{1}{e^{\frac{1}{T}\left(E_{0 p}-l \Omega-\mu\right)}-1} \\
& =\sum_{l=0}^{N} \int \frac{d p}{2 \pi} \frac{1}{e^{\frac{1}{T}\left(E_{0 p}+l \Omega+\mu\right)}-1} .
\end{aligned}
$$

This equation is solved by inspection with $\mu=-(N \Omega / 2)$. Therefore, the orbital assignments $l=N-m$ and $l=m$ for $\pi^{+}$and $\pi^{-}$in the LLL will have the same occupation number

$$
\begin{aligned}
n_{\pi^{+}}(l=N-m) & =n_{\pi^{-}}(l=m) \\
& =\int \frac{d p}{2 \pi} \frac{1}{e^{\frac{1}{T}\left(E_{0 p}-N \Omega / 2+l \Omega\right)}-1},
\end{aligned}
$$

with $0 \leq m \leq N$. For $N \Omega>2 m_{0}$, simultaneous condensation occurs for $m=0$; i.e., $\pi^{+}$with $l=N$ and $\pi^{-}$with $l=0$. For $(N-2) \Omega>2 m_{0}$, the condensation involves both $m=0,1$. As we increase $\Omega$ such that $\Omega=2 m_{0}$, all $m \leq(N / 2)$ will condense; i.e., $\pi^{+}$with $(N / 2) \leq l \leq N$ and $\pi^{-}$with $0 \leq l \leq(N / 2)$, and so on.

Now, consider the rotating ground state with $T=0$ and $N \Omega>2 m_{0}$ but $(N-2) \Omega<2 m_{0}$, so that only the $l=N$ state for $\pi^{+}$and the $l=0$ state for $\pi^{-}$condense. The energy per unit length in the Bose-Einstein condensate (BEC) state is

$$
\mathcal{E}_{\pi \Omega}=-\mathbf{n}\left(N \Omega-2 m_{0}\right)+d_{N} \mathbf{n}^{2},
$$

with the Coulomb factor

$$
d_{N} \approx \frac{e^{2}}{2} \int_{l_{M}}^{R} 2 \pi r d r\left(\frac{1}{2 \pi r}\right)^{2}=\frac{e^{2}}{4 \pi} \ln \frac{R}{a} \approx \frac{e^{2}}{8 \pi} \ln N .
$$

$d_{N}$ characterizes the electric field energy stored between two charged rings with radius $l_{M} \sim 1 / \sqrt{e B}$ and charge $-e$ $\left(\pi^{-}\right)$, and radius $R \gg l_{M}$ and charge $+e\left(\pi^{+}\right)$. The Coulomb self-energy is subleading and omitted. In the ground state, the BEC density $\mathbf{n}$ is fixed by minimizing the energy density $\mathcal{E}_{\pi \Omega}$ in (18), with the result

$$
\mathbf{n}=\theta\left(N \Omega-2 m_{0}\right) \frac{N \Omega-2 m_{0}}{2 d_{N}} .
$$

The rotating $\pi^{+}$condensate induces a uniform magnetic field $\mathbf{b}_{z}$ that enhances the applied initial field $B$ and backreacts on the formation of the charged condensates to order 
$\alpha=e^{2} / 4 \pi$. Indeed, the rotating BEC of $\pi^{+}$at $r=R$ generates an azimuthal current

$$
J^{\theta}[\mathbf{n}]=\frac{e \mathbf{n} N}{m_{0} r}\left|f_{0 N}\right|^{2} \approx \frac{e^{2} B \mathbf{n}}{4 \pi m_{0}} \delta(r-R),
$$

where $f_{0 N}$ is the LLL with angular momentum $l=N$. The corresponding induced magnetic field

$$
\mathbf{b}_{z}[\mathbf{n}]=\frac{e^{2} B \mathbf{n}}{4 \pi m_{0}}
$$

modifies the applied magnetic field to order $\alpha=e^{2} / 4 \pi$ through $B \rightarrow B+\mathbf{b}_{z}[n]$. The back-reacted LL problem amounts to the following substitutions for $m_{0}$ and $N$ :

$$
\begin{aligned}
m_{0}^{2}[\mathbf{n}] & =m_{\pi}^{2}+e B\left(1+\frac{e^{2} \mathbf{n}}{4 \pi m_{0}}\right), \\
N[\mathbf{n}] & =N\left(1+\frac{e^{2} \mathbf{n}}{4 \pi m_{0}}\right) .
\end{aligned}
$$

The back-reacted density for the $\pi^{ \pm}$condensates follows by minimizing the energy per unit length:

$$
\begin{aligned}
\mathcal{E}[\Omega, \mathbf{n}]= & -\mathbf{n}\left(N[\mathbf{n}] \Omega-2 m_{0}[\mathbf{n}]\right) \\
& +\mathbf{n}^{2} e^{2}\left(\frac{e B N}{16 \pi m_{0}^{2}[\mathbf{n}]}+\frac{\ln N[\mathbf{n}]}{8 \pi}\right) .
\end{aligned}
$$

This is the analogue of Eq. (18) with $d_{N}=\left[e^{2} \ln N(\mathbf{n}) / 8 \pi\right]$, including the additional magnetic energy from the backreaction

$$
\pi R^{2} \frac{\mathbf{b}_{z}^{2}}{2}=\frac{\mathbf{n}^{2} e^{4} B^{2} R^{2}}{32 \pi m_{0}^{2}[\mathbf{n}]}=\frac{e^{3} B N \mathbf{n}^{2}}{16 \pi m_{0}^{2}[\mathbf{n}]} .
$$

The true ground state follows by minimizing (24) with respect to $\mathbf{n}$. Both $m_{0}[\mathbf{n}]$ and $N[\mathbf{n}]$ are observed to be weakly dependent on the $\mathbf{n}$ contributions from the backreaction. We now explore the physical implication of Eq. (24) in heavy ion collisions.

Pion BEC in heavy ion collisions. - Current heavy ion collisions at collider energies are characterized by large angular momenta $l \sim 10^{3}-10^{5} \hbar$ [11] and large magnetic fields $B \sim m_{\pi}^{2}$ [12] in off-central collisions. While maintaining these strong fields all the way across the collision would be in general unexpected, even in the presence of a strong Faraday effect, we will assume here that they are still sizable at chemical freeze-out, where $R \sim 10 \mathrm{fm}$ with $e B \sim m_{\pi}^{2}$. This would translate to a LL degeneracy $N=$ $e B R^{2} / 2 \sim\left(m_{\pi} \times 10 \mathrm{fm}\right)^{2} \sim 100 / 4$ and a rotational chemical potential $\mu_{N}=N \Omega \sim 1.25 m_{\pi}$. From the hadrochemistry analysis, the pion chemical potentials at freeze-out are typically $\mu_{f} \sim 0.5 m_{\pi}$ at RHIC, and $\mu_{f} \sim 0.86 m_{\pi}$ at the LHC [14]. With the rotation at finite $B$, they would translate to $\mu_{\pi}=\mu_{N}+2 \mu_{f} \sim 1.96 m_{\pi}$ and $2.98 m_{\pi}$, respectively. Since the threshold of the LLL for the combined $\pi^{ \pm}$pion energy

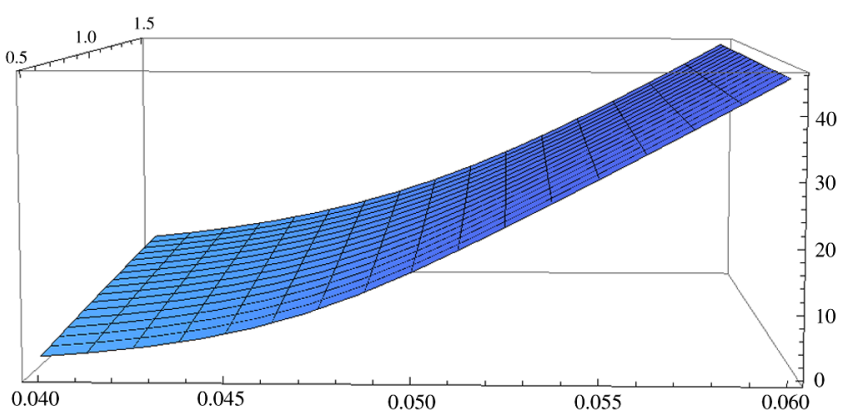

FIG. 2. The mean number of condensed pions $\mathbb{N}_{\pi^{+}}=\mathbb{N}_{\pi^{-}}$in the range $0.04 m_{\pi} \leq \Omega \leq 0.06 m_{\pi}$, for $\mu_{f}=0.86 m_{\pi}$ and $0.5 m_{\pi} \leq T \leq 1.5 m_{\pi}$.

is $2 \sqrt{2} m_{\pi}$, charge pion condensation is possible. Using (24) at finite $T, \mu_{f}$, the number of $\pi^{ \pm}$pions in the BEC is

$$
\mathbb{N}_{\pi^{ \pm}}=\frac{\sum_{n=0}^{\infty} n e^{-\frac{1}{T}\left(L \mathcal{E}\left[\Omega, \frac{n}{L}\right]-2 n \mu_{f}\right)}}{\sum_{n=0}^{\infty} e^{-\frac{1}{T}\left(L \mathcal{E}\left[\Omega, \frac{n}{L}\right]-2 n \mu_{f}\right)}}
$$

For $L \sim 10 \mathrm{fm}, e B \sim m_{\pi}^{2}$ and $N \approx 25$, we show in Fig. 2 the average number of condensed $\pi^{ \pm}$for temperatures in the range $0.5 m_{\pi} \leq T \leq 1.5 m_{\pi}$ and rotations in the range $0.04 m_{\pi} \leq \Omega \leq 0.06 m_{\pi}$ for the most favorable case with $\mu_{f}=$ $0.86 m_{\pi}$ at the LHC. It is interesting to note that the ALICE Collaboration has recently reported a large coherent emission from multipion correlation studies in $\mathrm{Pb}-\mathrm{Pb}$ collisions [13].

Conclusions. - The combined effects of a rotation parallel to a magnetic field yields to pion condensation both in the vacuum and at finite temperature. The $\pi^{+}$pions condense at the edge, while the $\pi^{-}$pions condense at the center in equal amounts when charge conservation is strictly enforced in a closed volume. Since parallel rotations and magnetic fields can be generated in current heavy ion collisions at collider energies, charged pion condensation could be generated if the combined effects survive with considerable strength in the freeze-out phase. Such effects are likely to affect both the flow of charge particles and their number fluctuations. This separation of charged bosons by centrifugation in a magnetic field may also be probed in atomic physics (trapped and cooled atoms), in condensed matter physics (quantum Hall effect) and possibly in compact stars (magnestars).

We thank Edward Shuryak for a discussion. This work was supported in part by the U.S. Department of Energy under Contract No. DE-FG-88ER40388.

*yizhuang.liu@stonybrook.edu ismail.zahed@stonybrook.edu

[1] A. Vilenkin, Macroscopic parity violating effects: Neutrino fluxes from rotating black holes and in rotating thermal radiation, Phys. Rev. D 20, 1807 (1979); Equilibrium parity 
violating current in a magnetic field, Phys. Rev. D, 22, 3080 (1980).

[2] S. Ebihara, K. Fukushima, and T. Oka, Chiral pumping effect induced by rotating electric fields, Phys. Rev. B 93, 155107 (2016).

[3] D. E. Kharzeev, K. Landsteiner, A. Schmitt, and H. U. Yee, Strongly interacting matter in magnetic fields: An overview, Lect. Notes Phys. 871, 1 (2013).

[4] Y. Liu and I. Zahed, QCD-inspired Dirac spectrum at finite chemical potential: Anomalous effective action, Berry phase and composite fermions, arXiv:1509.00812.

[5] K. Hattori and Y. Yin, Charge Redistribution from Anomalous Magnetovorticity Coupling, Phys. Rev. Lett. 117, 152002 (2016).

[6] Y. Jiang and J. Liao, Pairing Phase Transitions of Matter under Rotation, Phys. Rev. Lett. 117, 192302 (2016).

[7] S. Ebihara, K. Fukushima, and K. Mameda, Boundary effects and gapped dispersion in rotating fermionic matter, Phys. Lett. B 764, 94 (2017); H. L. Chen, K. Fukushima, X. G. Huang, and K. Mameda, Analogy between rotation and density for Dirac fermions in a magnetic field, Phys. Rev. D 93, 104052 (2016).

[8] M. N. Chernodub and S. Gongyo, Interacting fermions in rotation: Chiral symmetry restoration, moment of inertia and thermodynamics, J. High Energy Phys. 01 (2017) 136; Effects of rotation and boundaries on chiral symmetry breaking of relativistic fermions, Phys. Rev. D 95,
096006 (2017); Edge states and thermodynamics of rotating relativistic fermions under magnetic field, Phys. Rev. D 96, 096014 (2017).

[9] F. Becattini, F. Piccinini, and J. Rizzo, Angular momentum conservation in heavy ion collisions at very high energy, Phys. Rev. C 77, 024906 (2008).

[10] Y. Jiang, Z. W. Lin, and J. Liao, Rotating quark-gluon plasma in relativistic heavy ion collisions, Phys. Rev. C 94, 044910 (2016); Erratum, Phys. Rev. C 95, 049904(E) (2017); W. T. Deng and X. G. Huang, Vorticity in heavyion collisions, Phys. Rev. C 93, 064907 (2016).

[11] L. Adamczyk et al. (STAR Collaboration), Global $\Lambda$ hyperon polarization in nuclear collisions: Evidence for the most vortical fluid, Nature (London) 548, 62 (2017).

[12] D. E. Kharzeev, J. Liao, S. A. Voloshin, and G. Wang, Chiral magnetic and vortical effects in high-energy nuclear collisions: A status report, Prog. Part. Nucl. Phys. 88, 1 (2016).

[13] J. Adam et al. (ALICE Collaboration), Multipion BoseEinstein correlations in $p p, p-\mathrm{Pb}$, and $\mathrm{Pb}-\mathrm{Pb}$ collisions at energies available at the CERN large Hadron Collider, Phys. Rev. C 93, 054908 (2016).

[14] V. Begun, Fluctuations as a test of chemical non-equilibrium at the LHC, Phys. Rev. C 94, 054904 (2016).

[15] G. E. Volovik, The Universe in a Helium Droplet (Oxford University Press, New York, 2003).

[16] Y. Liu and I. Zahed, Rotating Dirac fermions in a magnetic field in $1+2,3$ dimensions, arXiv:1710.02895. 